\title{
Substance Abuse Treatment: The Case for Adaptive Interventions
}

\author{
Keith $\mathrm{K}^{*}$, Theresa $\mathbf{M}$ and Emma $\mathbf{P}$ \\ USA \\ *Corresponding author: Keith K, USA

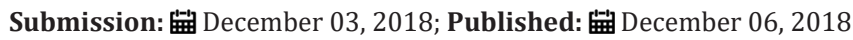

\section{Introduction}

The call for investigators is to develop flexible substance abuse treatments, which can be modified, based on clients/ couples presenting issues or concerns, in contrast to the one-sizefits-all approach typically developed and tested in clinical trials. Unfortunately, dissemination has become a major obstacle for researchers and treatment developers alike in that empirically supported models are not being widely adopted in community settings. Thus, there is a need to identify strategies for modifying and delivering these interventions to allow for easier and more widespread implementation into community practice, while not comprising the integrity of these treatments. To that end, clinicians and researchers alike may wish to shift the focus of treatment from the macro (empirically supported treatments applied across specific conditions regardless of unique client differences) to the micro (practice-based evidence based on individual progress reported on an ongoing basis).

From a practice-based evidence vantage, manuals and session material may be best viewed as a set of guiding principles rather than prescriptive procedures. Conceptualizing treatment in this manner aids in dissemination since it provides therapists more flexibility as well as creativity in delivering the intervention and is the way therapy is typically conducted in community-based settings; treatment is modified based on changes to the client's presenting issues or concerns. That said, the next phase in the evolution of evidence-based treatments is to develop adaptive interventions that take into account client-level variability in terms of progress and allows for changes to duration, intensity, and skills emphasized to accommodate patient needs. Adaptive interventions involve using decision rules to modify the intensity or type of treatment based on the client's needs.

\section{Behavioral Couples Therapy for Substance Use Disorders}

Behavioral Couples Therapy for Substance Use Disorders (BCTSUD) is an empirically supported, manualized 12-session treatment for alcohol and substance misuse that concurrently addresses both relationship and substance use problems. BCT-SUD assumes that the substance misuse and relational problems are interrelated and improvements in each area can serve as a resource for the client's recovery and overall family functioning. Despite extensive empirical support for its efficacy compared to individual-based treatment, it has not been widely adopted in community practice, in part, due to the perceived rigidity in which it can be applied.

Although some clients may benefit from the full course of treatment (i.e., 12 sessions), others may not need the entire 12 sessions or in fact, may need more with particular emphasis on specific relational (e.g. communication, conflict resolution) or substance use components (e.g. continuing recovery). Given that the knowledge base about the efficacy of BCT-SUD is well established, the next direction in this programmatic line of research is to identify ways to contextualize the intervention to more precisely meet clients' needs so it is more akin to treatment delivery in realworld settings. Moreover, contextualizing the treatment to meet individual couples' needs may also result in improved retention rates since outcomes are more regularly monitored and treatment modified based on data (rather than all couples receiving 12 sessions) and potentially improved clinical and cost outcomes.

Collins et al. [1] have developed methodologies for translating existing treatments into adaptive interventions. Sequential, Multiple Assignment, Randomized Trials (SMART) involves multiple stages with stage corresponding to critical decisions involved in the adaptive intervention. At each stage, all participants are randomly assigned to one of the treatment options. By randomizing participants multiple times, scientists can assess the effectiveness of each option and then develop the best set of decision rules (Methodology Center, Penn State). SMART provides data that can guide the construction of adaptive interventions. Once the adaptive intervention has been completed, the delivery of the adaptive treatment is not randomized Lei et al. [2].

\section{Conclusion}

Despite the overwhelming support for BCT, this treatment approach has not been widely adopted by community providers. Part of the dissemination issues may be related to the fact that the treatment is not consistent with the manner in which treatment is conducted in the real world. The development of an adaptive BCT approach that retained the efficacy of the standard version but is 
flexible enough to be modified according to patient progress would provide practitioners an empirically supported treatment that could be easily disseminated to community providers.

\section{References}

1. Collins LM, Nahum SI, Almirall D (2014) Optimization of behavioral dynamic treatment regimens based on the sequential, multiple assignment, randomized trial (SMART). Clinical Trials 11(4): 426-434.
2. Lei H, Nahum SI, Lynch K, Oslin D, Murphy SA (2012) A "SMART" design for building individualized treatment sequences. Annual Review of Clinical Psychology 8: 21-48.
Creative Commons Attribution 4.0

International License

For possible submissions Click Here

Submit Article

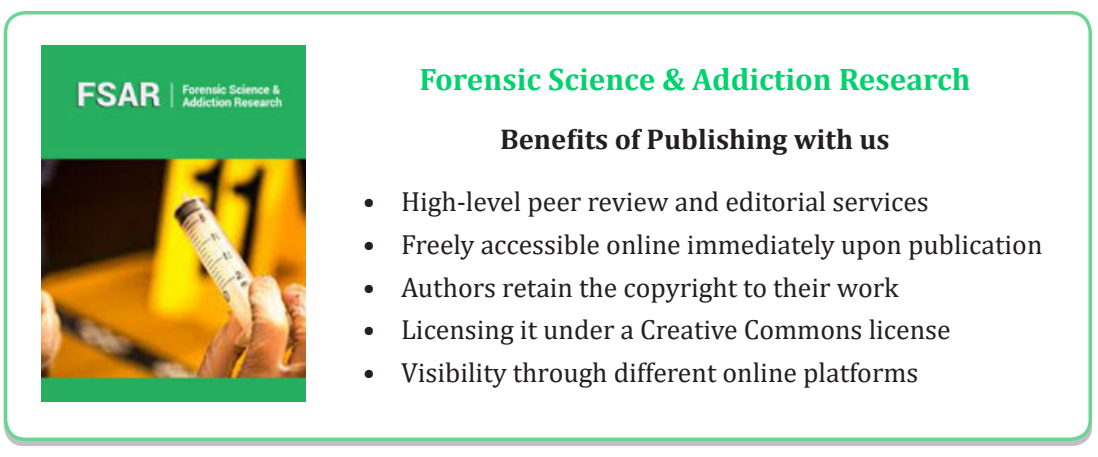

